\title{
TeV Gamma-ray Astronomy: The Story So Far
}

\author{
Trevor C. Weekes \\ Fred Lawrence Whipple Observatory, Harvard-Smithsonian Center for Astrophysics, Amado, AZ 85645, USA \\ tweekes@cfa.harvard.edu
}

\begin{abstract}
A snapshot is presented of the present status of our knowledge of the TeV gamma-ray universe. Emphasis is put on observations made using the imaging atmospheric Cherenkov technique. The capabilities of the present generation of telescopes is listed. Progress has been dramatic and several features have been different from what was anticipated. The catalog of sources includes some 78 objects and these are tabulated as extragalactic sources (24), supernovae remnants (11), pulsar wind nebulae (10), binaries (4), miscellaneous (9), diffuse high energy sources (3) and unidentified sources (20). Some comments are made on the factors influencing the past and future development of the field.
\end{abstract}

Keywords: $\mathrm{TeV} \gamma$-Ray Observations

PACS: $97.60 . \mathrm{Bw}$, 97.60.Gb, 97.60.Lf, 98.70.Sa, 98.54.Cm

\section{STATUS OF TEV GAMMA-RAY ASTRONOMY, C. 2008}

Having had the honor of presenting status reports at two of the last three Heidelberg meetings on TeV gamma-ray astronomy [1], [2], I am painfully aware (with the benefit of hindsight) of my shortcomings at such a task, in particular at my attempts to predict the future growth of the field where I have generally erred on the conservative side and have been pleasantly surprised by the pace of discovery.

Hence here I will confine myself to reporting on the status of the field as I know it today (July, 2008) which will therefore rely mostly on my knowledge of the published papers and recent excellent reviews [3], [4], [5] as well as the comprehensive White Paper on $\mathrm{TeV}$ groundbased gamma-ray astronomy that was recently put together by members of the US TeV gamma-ray community [6]. I will attempt to summarize the status and capability of the various observatories, outline the sensitivity that can be achieved with existing instrumentation, the range of observed phenomena, make some attempt to catalog the credible discrete sources reported to date, and provide some personal perspective on the progress of, and prospects for, the field. I will make no attempt to describe the significant improvements that are planned for existing observatories, some of which are in an advanced state of construction.

\section{Caveat}

Nothing dates more rapidly than a written status report. This is particularly true of one presented at the beginning of a symposium where the most exciting results from the various groups have been embargoed so that they can be presented at the symposium.

Thus even before the ink on the report is dry, it is outof-date. Its value therefore is merely to serve as a historical benchmark and to summarize what one of us thought he knew at the start of the symposium and to provide some kind of reference point for future developments.

\section{WHAT WE HAVE TODAY}

\section{The Atmospheric Cherenkov Technique}

With the notable exception of the remarkable results from the Milagro experiment [7], the bulk of the observational results at $\mathrm{TeV}$ energies have come from telescopes using the atmospheric Cherenkov technique. Although the basic technique was developed some fifty years ago, it was not until the development of the so-called imaging atmospheric Cherenkov technique (IACT) that the first indication of a credible detection was apparent [8], [9]. Given the rather murky history that has characterized the early results at all gamma-ray energies (100 MeV energies as well as $1 \mathrm{TeV}$ energies), it is not surprising that this early detection was treated with some skepticism.

In its earliest manifestation the technique was characterized by its:

- Simplicity

- Economy

- Elegance

Although the early experiments (an example, the first Whipple Observatory experiment shown in Figure 1) did not succeed in producing convincing evidence for the existence of any sources, they showed that it was possible 
for small groups to participate in the exciting new field of high energy astrophysics; this field was soon to be dominated by elaborate and expensive experiments in balloons or on satellites. The elegance of the Cherenkov technique was apparent in its economy as to the energy intercepted that was necessary to detect the gamma ray; as Ken Greisen pointed out, the ground-based technique is remarkable in that only one millionth of the energy of the primary gamma ray (in the form of Cherenkov light photons) need be collected by the telescope for the gamma ray to be detected.

The imaging atmospheric Cherenkov technique as practiced today (Figure 2) with its multitude of pixels, multiple large optical reflectors, and high speed data acquisition systems is certainly not simple. Of necessity, the costs of such systems are now large and prohibitive for small research programs. The typical state-of-the-art observatory costs $\$ 20 \mathrm{M}$ and authorship lists are in the hundreds. Only the elegance survives and still makes the technique attractive to cosmic ray physicists and refugees from large high energy physics experiments.

With the current emphasis on "green" technology it is perhaps worth noting that the detection of high energy particles using their secondary emissions in the atmosphere is inherently a "green" technique since in no way is the natural path of the gamma ray, destined to cross the wilderness of interstellar and perhaps intergalactic space and end its life by collision with an air molecule, disturbed. The gamma ray is not even aware it has been detected! In contrast the highly technical gamma-ray space telescope must intercept, and destroy, the primary gamma ray in its complex silicon layers; it thus ends its life prematurely and catastrophically and never completes its original destiny.

\section{The Tools Available Today}

Although all ground-based experiments that have the sensitivity to detect a source like the Crab Nebula can contribute to $\mathrm{TeV}$ gamma-ray astronomy, the recent literature (and this symposium) has tended to be dominated by the "Big Three", H.E.S.S., MAGIC and VERITAS. However CANGAROO III, the Whipple 10m telescope and the recently completed HAGAR in Ladhak, India also play an important role, particularly in monitoring variable sources. Some of the characteristics of these observatories are listed in Table 1.

It should be noted that only three of the original four telescopes of CANGAROO III are now in operation so the sensitivity is reduced from the original designed threshold (M. Mori, private communication). The threshold shown for H.E.S.S. is that at first light and when most of their pioneering discoveries were made; because of mirror weathering the threshold is now higher. HAGAR has only recently come on-line so its sensitivity has still to be confirmed; this value and those for the other two Indian experiments were supplied by B.S. Acharya (private communication). HAGAR is noteworthy in that it will be the first telescope to operate at an elevation $>4 \mathrm{~km}$. Of the eight observatories listed, all but HAGAR and PACT use the IACT; their approach is to use wavefront sampling with an array of small telescopes.

\section{THE PRESENT CAPABILITIES}

\section{Wide Spectral Energy Range : $25 \mathrm{GeV}$ to $100 \mathrm{TeV}$}

The earliest experiments had energy thresholds in excess of $5 \mathrm{TeV}$. As the size of the telescopes has increased and the sophistication of the triggering improved, the energy threshold has steadily dropped, so that now results are presented with thresholds as low as $25 \mathrm{GeV}$ (Figure 3 ). The upper energy bound is determined by exposure time and can be extended by observing at low elevations where the collection area and energy threshold increase. It is certainly possible to make observations with telescopes using the IACT up to energies of $100 \mathrm{TeV}$ (Figure 4, Figure 5). The motivation to go to lower energies comes mainly from the desire to study distant AGN, which are expected to have soft spectra, and Gamma Ray Bursts and pulsars for the same reason. Higher energies are particularly important in the study of Supernovae Remnants since such observations have the best hope of separating out the contributions from hadronic and electronic progenitors. Although most IACT observatories strive to achieve the lowest possible energy thresholds, in practice the technique is still most sensitive at energies around $200 \mathrm{GeV}$ and this is where most of the new sources have been discovered. The detection of the Crab pulsar (this symposium) is an obvious exception.

\section{Energy resolution: 10 to $35 \%$}

Once a source has been detected, interest centers on the determination of the energy spectrum. Most sources can be fit with a simple power law. Structure in the spectrum can be an important clue to the emission mechanism and possible absorption processes. Energy resolution can range from as little as $10 \%$ with arrays of telescopes, which permit the determination of the impact parameter, to $30-40 \%$ with single telescopes. The signal strength should be at least $5 \sigma$ for a meaningful measurement. At low energies spectral mesurements are limited by threshold effects and at high energies by statistics. A 


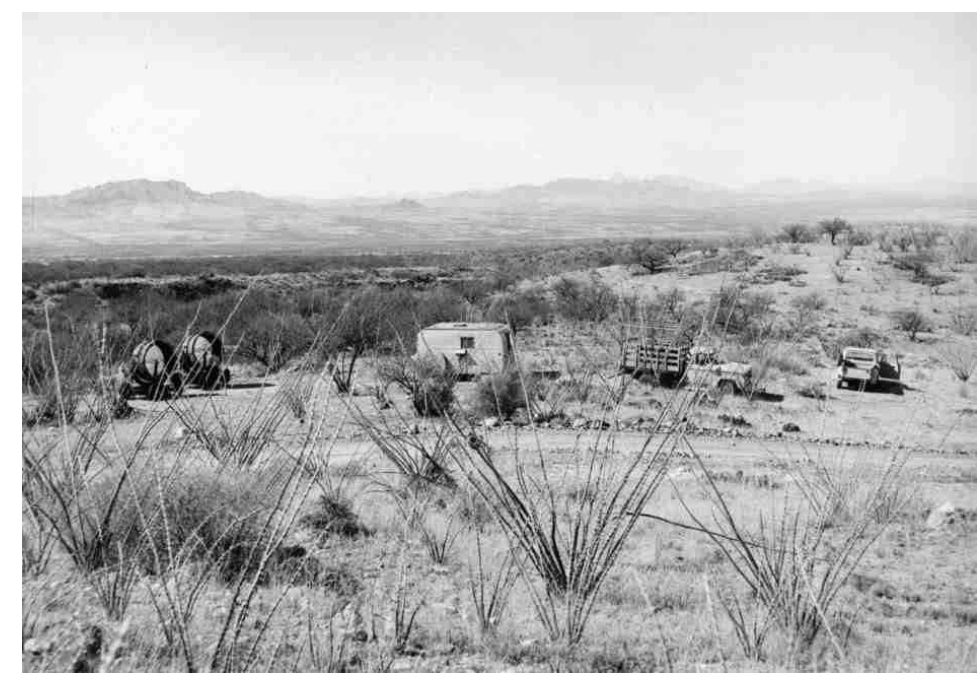

FIGURE 1. The first TeV gamma-ray observatory in the United States consisted of two 1.5m telescopes (made from World War II searchlight reflectors) above (left center); the telescopes were manually operated and were located at a dark site in southern Arizona during the winter of 1967-8 [10]. The telescopes were directed (by eye) at a point ahead of the position of the putative source so that the earth's rotation swept the source through the field of view. Power came from an electric generator on the back of the truck (center right) and the pulse counting electronics were housed in a small trailer (center). The system was mercifully free of computers and the analysis was done offline with a mechanical calculator. No sources were detected.

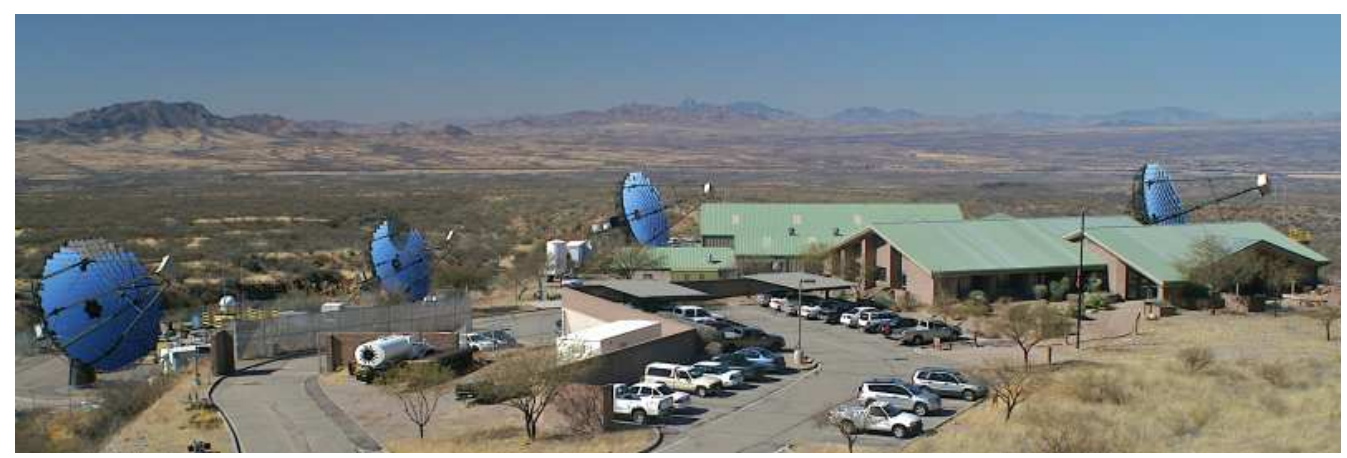

FIGURE 2. The VERITAS observatory, the newest of the third generation IACT observatories, saw first light in April, 2007. Note that VERITAS is in the exact same location as the telescopes shown in Figure 1. Each of the four telescopes has an aperture of $12 \mathrm{~m}$ (collection area of $106 \mathrm{~m}^{2}$ ) and a camera with 499 pixels.

TABLE 1. Major Existing ACT Facilities

\begin{tabular}{lrrrrrrr}
\hline \multicolumn{1}{c}{ Observatory } & $\begin{array}{r}\text { Elevation } \\
(\mathbf{k m})\end{array}$ & $\begin{array}{r}\text { Telescopes } \\
\#\end{array}$ & $\begin{array}{r}\text { Mirror Area } \\
\left(\mathrm{m}^{2}\right)\end{array}$ & $\begin{array}{r}\text { FoV } \\
(\mathbf{d e g r e e s})\end{array}$ & First Light & $\begin{array}{r}\text { Threshold } \\
(\mathbf{G e V})\end{array}$ & $\begin{array}{r}\text { Sensitivity } \\
(\boldsymbol{\%} \mathbf{C r a b})\end{array}$ \\
\hline H.E.S.S. & 1.8 & 4 & 428 & 5 & 2003 & 100 & 0.7 \\
VERITAS & 1.3 & 4 & 424 & 3.5 & 2007 & 100 & 1 \\
MAGIC & 2.2 & 1 & 236 & 3.5 & 2005 & 50 & 1.6 \\
HAGAR & 4.3 & 7 & 31 & 3 & 2008 & 60 & 9 \\
Whipple & 2.3 & 1 & 75 & 2.2 & 1985 & 400 & 10 \\
CANGAROO III & 0.1 & $3(4)$ & $172(230)$ & 4 & 2006 & 400 & 10 \\
PACT & 1.1 & 24 & 107 & 3 & 2001 & 750 & 11 \\
TACTIC & 1.3 & 1 & 10 & 2.8 & 2001 & 1500 & 70 \\
SHALON & 3.3 & 1 & 11.2 & 8 & 1996 & $1000 ?$ & $?$ \\
\hline
\end{tabular}




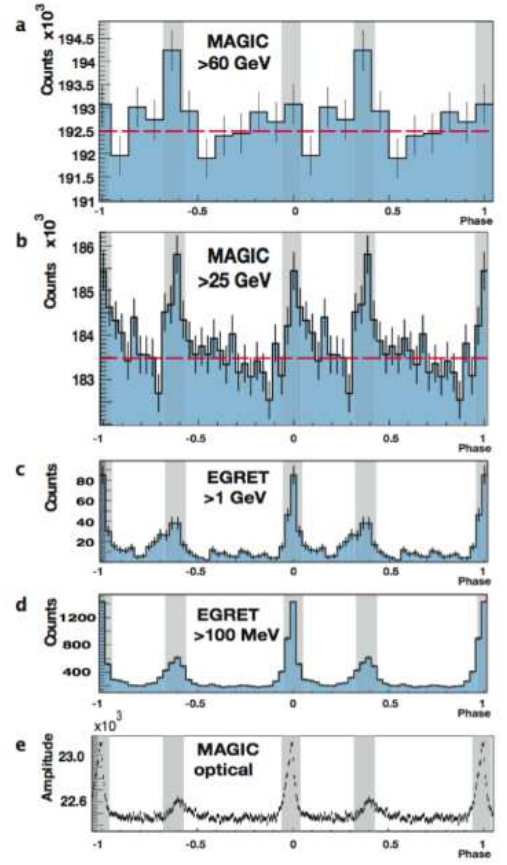

FIGURE 3. The first indication of a weak pulsed signal from the Crab pulsar at energies above $60 \mathrm{GeV}$ from the MAGIC group [11]. A later report (this symposium) confirmed the signal and extended the observations down to $25 \mathrm{GeV}$, the lowest energy at which a signal has been reported using the atmospheric Cherenkov technique.

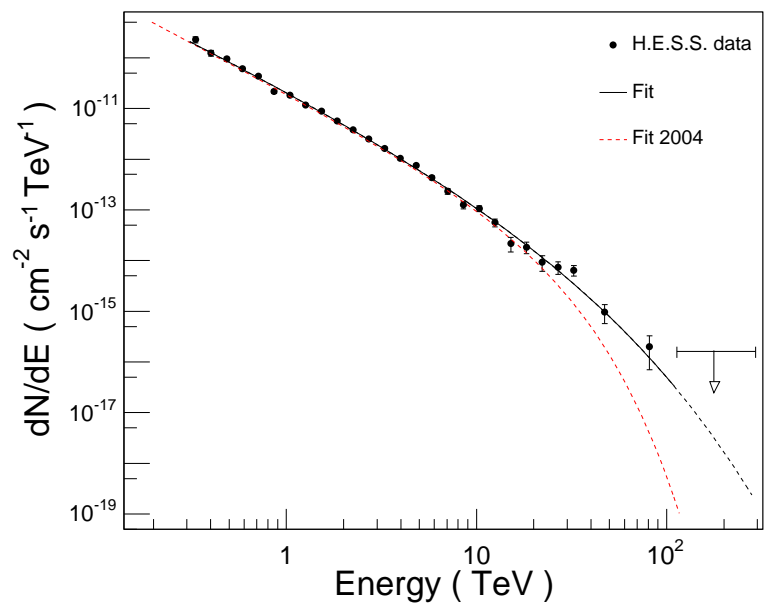

FIGURE 4. The H.E.S.S. gamma-ray spectrum of RX J1713.7-3946, the strongest gamma-ray source in the Southern Hemisphere [12]. The data points can be fit by a power law with exponential cutoff. The upper limit, indicated by the black arrow, covers the energy range from 113 to $300 \mathrm{TeV}$. Particle acceleration up to at least $100 \mathrm{TeV}$ is inferred from these observations; although the progenitor particles could be hadrons, electron progenitors are not ruled out.

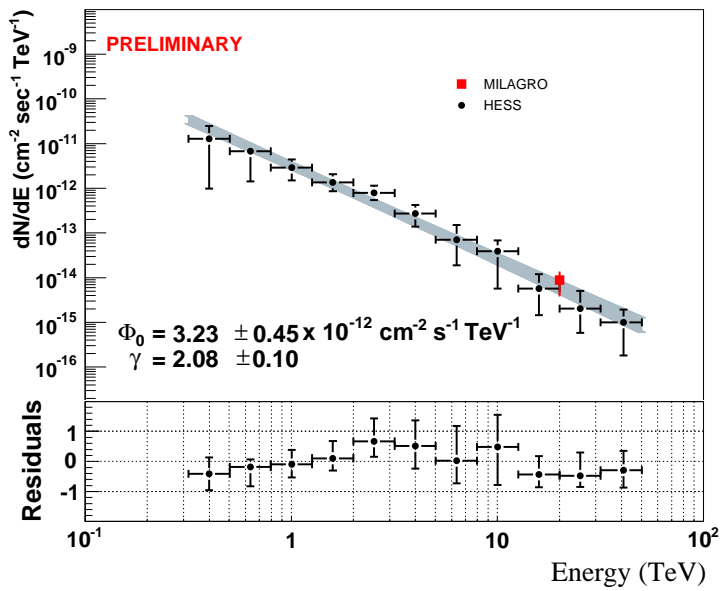

FIGURE 5. The differential energy spectrum of the extended source which was first reported by Milagro [7]. The spectrum shown here is as detected by H.E.S.S. [13] which saw it as a hard spectrum source above $300 \mathrm{GeV}$. The H.E.S.S. source is extended, with a FWHM size of $0.5^{\circ}$. The single differential point seen by Milagro at $20 \mathrm{TeV}$ is shown as a square.

steep spectrum must always be treated with caution since this is the characteristic of a false detection which is usually caused by an uneven sky background effect.

Energy resolution is important to those who see $\mathrm{TeV}$ astronomy as a window in which to explore dark matter; a source with high density might be expected to have a high concentration of neutralinos and might be identified by a line in the $100-1000 \mathrm{GeV}$ range. This possibility is a driving force for many $\mathrm{TeV}$ scientists with a background in high energy physics.

\section{Flux Range: 1 to $1500 \%$ of the Crab}

The Crab Nebula is the strongest steady $\mathrm{TeV}$ source in the sky; with a declination of $+22^{\circ}$ it is visible from both hemispheres and has a moderately hard spectrum. Thus it is useful as a standard candle for comparing instrument sensitivities and source strengths. With integration times of 50 hours (a sizable fraction of the observing year for most observatories), sources can be detected that are $1 \%$ of the Crab (Figure 6). However most of the reported sources have signal strengths well in excess of $1 \%$. Systematics tend to limit longer integration times and hence the detection of weaker sources. Flaring AGN have been detected with fluxes (for short periods) in excess of fifteen times the Crab (Figure 7).

The usual standard for acceptance of a new source is that the signal should be at the $5 \sigma$ level. This is a fairly conservative criteria and is probably justified when the systematics are not fully understood. However observa- 


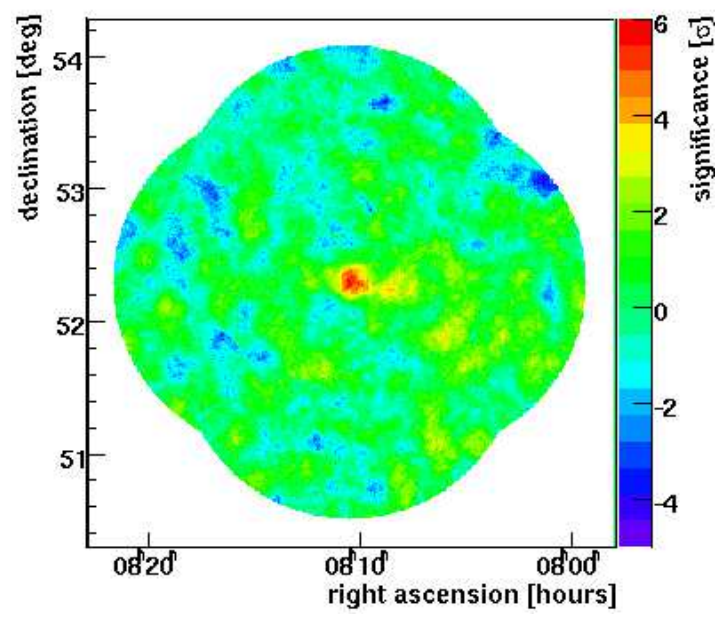

FIGURE 6. VERITAS observations of the HBL blazar $1 \mathrm{ES} 0806+514(\mathrm{z}=0.138)$ show weak but steady emission above $300 \mathrm{GeV}$ [14]; [15]. Observations were carried out during the construction of VERITAS and incorporate data using two, three and four telescopes. This is an example of a detection at approximately $1 \%$ of the Crab Nebula flux.

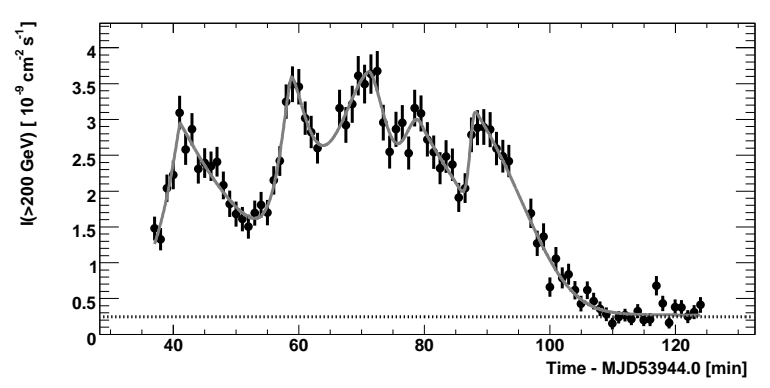

FIGURE 7. The extraordinary outburst from PKS2155-302 observed by H.E.S.S. in 2006 [16]. At peak the flux was 15 times that of the Crab Nebula. Short-term variability has also been seen from other AGN: Markarian 421 by Whipple and VERITAS and Markarian 501 by MAGIC. The time-scales of these bursts are among the fastest ever seen in blazars at any wavelength.

tions of important candidate objects which are above the $4 \sigma$ level should certainly be reported but treated with some caution. Independent verification by another observatory is perhaps the best criterion for credibility but if that standard was adopted the $\mathrm{TeV}$ source list would be very short. This is particularly so in the Southern Hemisphere where most of the sources have been seen by just one experiment.

\section{Angular Resolution: 2 arc-min to 3 degrees}

The IACT is optimized for point source detection. H.E.S.S. has an angular resolution of 2 arc-min as demonstrated in the beautiful map of RX J1713.7-3946 (Figure 8). In general, extended sources are more difficult to detect by IACT telescopes. In the surveys that have been made of the Galactic Plane it is clear that many Galactic sources are not point-like. The Milagro experiment has poorer resolution but greater sensitivity for the detection of extended sources (Figure 5). The source location capability is usually sufficiently good that there is no ambiguity in the identification with the target object. Unlike the $100 \mathrm{MeV}$ region where the gamma-ray point source sensitivity is severely limited by the contribution from the Galactic Plane, TeV observations have basically the same sensitivity over all the sky and hence offer better opportunities for source identification for sources that are detected in both energy bands.

In some cases correlated time variability at other wavelengths with superior angular resolution can lead to source identification on the sub-arc-min scale, e.g. in M87 (Figure 9, Figure 10).

\section{Distance: 500 ly to 1 billion ly}

The closest source reported may be Geminga which was nominated as a candidate source by the Milagro group [7]. Blazars are the most distant objects (Figure 11) that have been detected. The most distant source detected is still somewhat controversial since there is always uncertaintly about the reshifts of BL Lac objects because of their paucity of emission lines. One would expect the observed spectra of AGN to soften with increasing redshift since the infrared absorption effect should increase with energy; this is generally the case (Figure 12). Certainly there are several AGN detected with redshifts in excess of 0.2 ; the most distant object is probably 3C279 but this detection still awaits confirmation. 3C66a, which was originally detected by the Crimean Astrophysical Observatory group [18] and has recently been confirmed by VERITAS [19], is reported to have a redshift of 0.444 .

Because the IACT has very good flux sensitivity for the detection of short transients, e.g. Gamma Ray Bursts, it may be that these will be the most distant detectable sources of $\mathrm{TeV}$ gamma rays.

\section{Time Variations: minutes to years}

Although some TeV sources have been observed to exhibit rapid time variability, the majority of the reported 


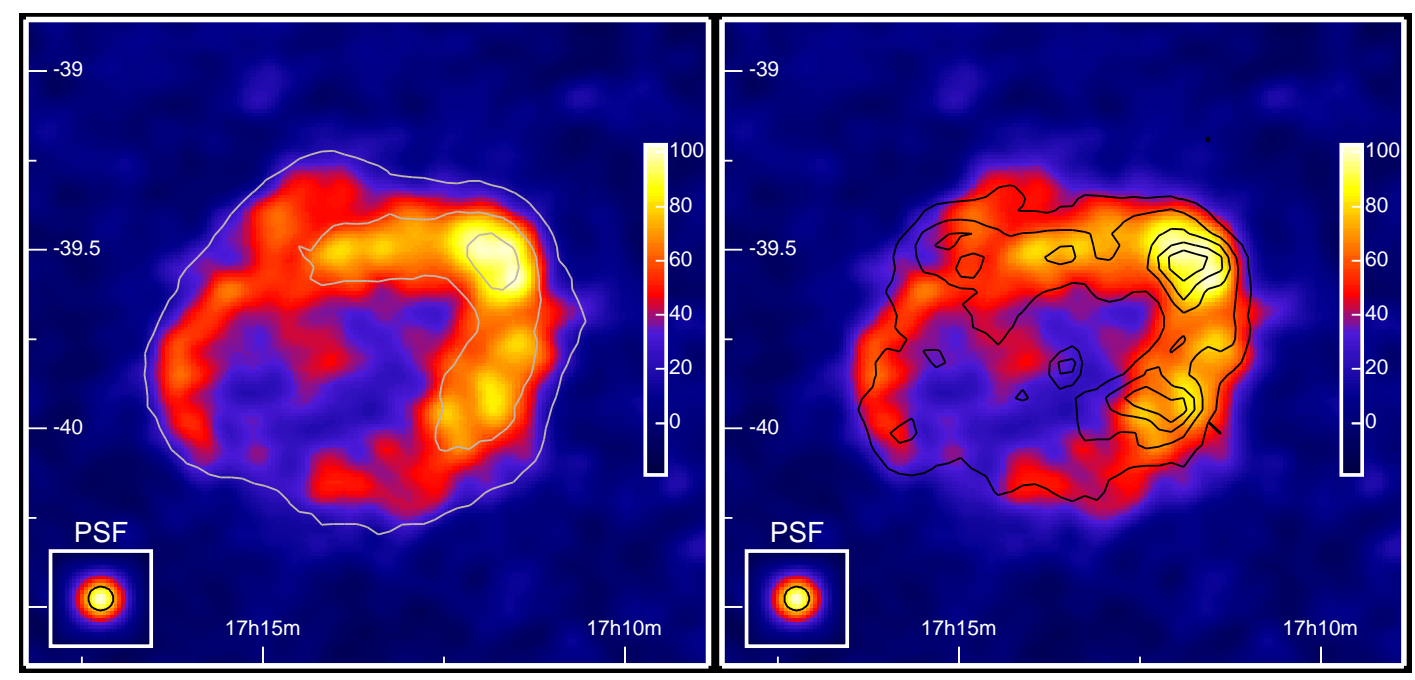

FIGURE 8. These are two remarkable representations of the H.E.S.S. observations of RX J1713.7-3946 [12]. The images are smoothed with a Gaussian of 2 arc-min. On the left, the overlaid light contours show the significance levels of the different features. The levels are at 8, 18, and $24 \sigma$. On the right the X-ray ASCA contours are shown as black lines. The full detail can only be seen in the color version of the figure in the original publication
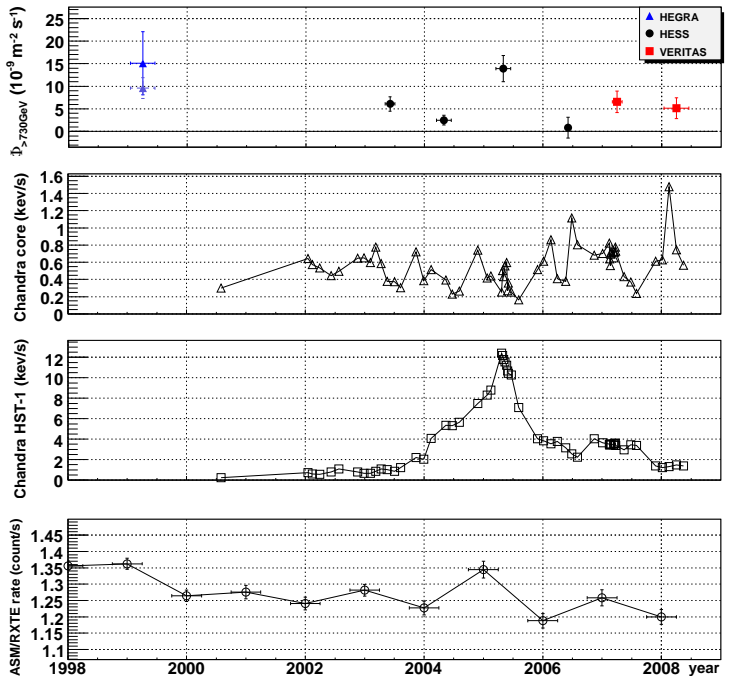

FIGURE 9. Top panel: the ten year light curve of M87 in $\mathrm{TeV}$ gamma rays and soft X-rays [17]. The TeV gamma-ray points are from HEGRA, H.E.S.S. and VERITAS. Second and third panels: X-ray data from Chandra (D. E. Harris, private communication) from the core and knot, HST-1. Bottom panel: X-ray data from the ASM/RXTE quick-look web page. Although this gamma-ray light-curve only shows the variations on a one year time-scale, the gamma-ray flux has also been seen to vary on a time scale of days.

$\mathrm{TeV}$ sources exhibit steady emission within the sensitivity limits of the observatories and the duration of the observations. Time variations have been observed on a scale as short as 2-3 minutes (Figure 7) and as long as

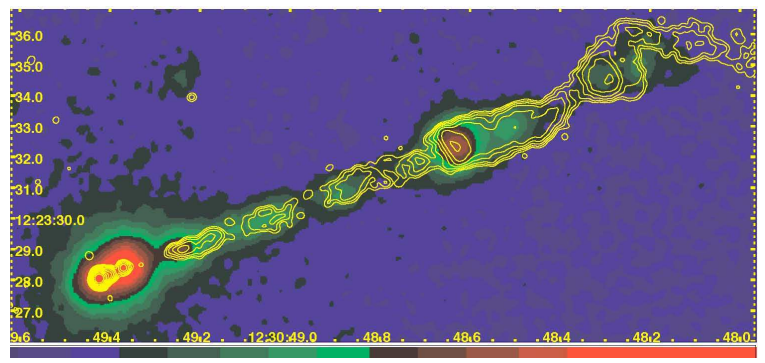

FIGURE 10. The X-ray image of the jet in M87 as seen by Chandra (D. Harris, private communication). Radio contours are superimposed. Interest has centered on the core of the object (bottom left hand corner) which is resolved in X-rays into two objects, the core and the close knot called HST-1. Initially it was thought that the $\mathrm{TeV}$ variations were correlated with the $\mathrm{X}$ ray emission from HST-1 (in 2005) but more recently it appears that it is correlated with the core.

years (Figure 9). Some sources are only detected when a flare occurs, e.g. W Comae (Figure 13). The variability of the AGN sources makes TeV astronomy more interesting; it is impossible to predict when a source like Markarian 421 will be flaring so that on-line data analysis is always exciting and often enough to keep the observer awake during a long night of observing. In a few cases optical brightening of the AGN has triggered the detection of TeV flaring AGN. The Galactic sources are generally steady and predictable; the exceptions are the periodic pulsar and binary sources.

The time-scale of the variations of the AGN signals limits the size of the emitting region and can be an important probe for cosmological and fundamental physics 


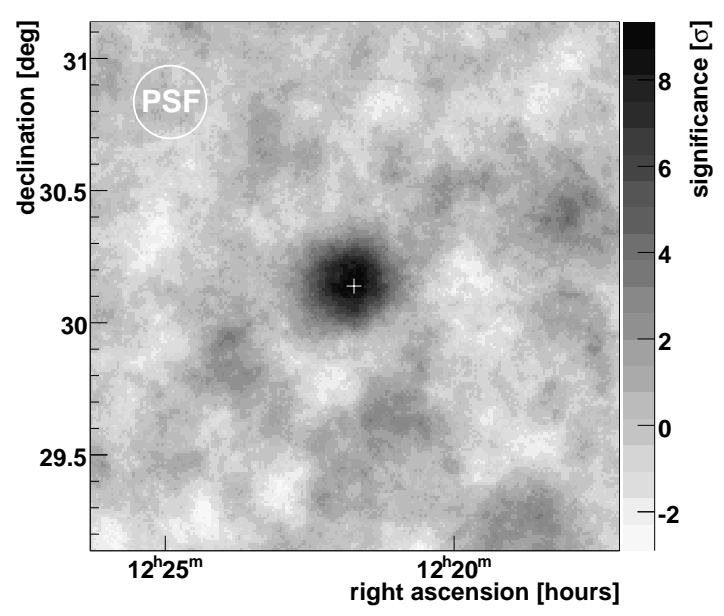

FIGURE 11. A map of the significances around the region of the distant HBL AGN, 1ES 1218+304 ( $\mathrm{z}=0.182)$ as seen by VERITAS [20]. The white cross indicates the position of the radio source. The white circle in the upper left corner shows the angular resolution of VERITAS (PSF).

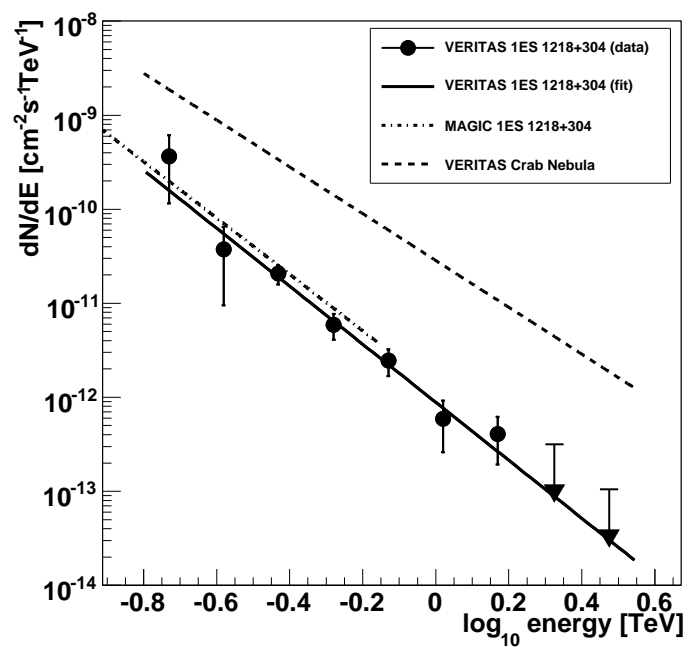

FIGURE 12. The differential energy spectrum of $1 E S 1218+304$ measured by VERITAS with a power law fit shown by the continuous straight line [20]. The dot-dash line shows the spectrum measured by MAGIC in their discovery observations [22]. The dash line is the spectrum of the Crab Nebula as measured by VERITAS.

studies. Doppler factors in excess of 100 are required to explain the observed variability if one assumes the emission region has a size comparable to the Schwarzschild radius of a massive black hole.
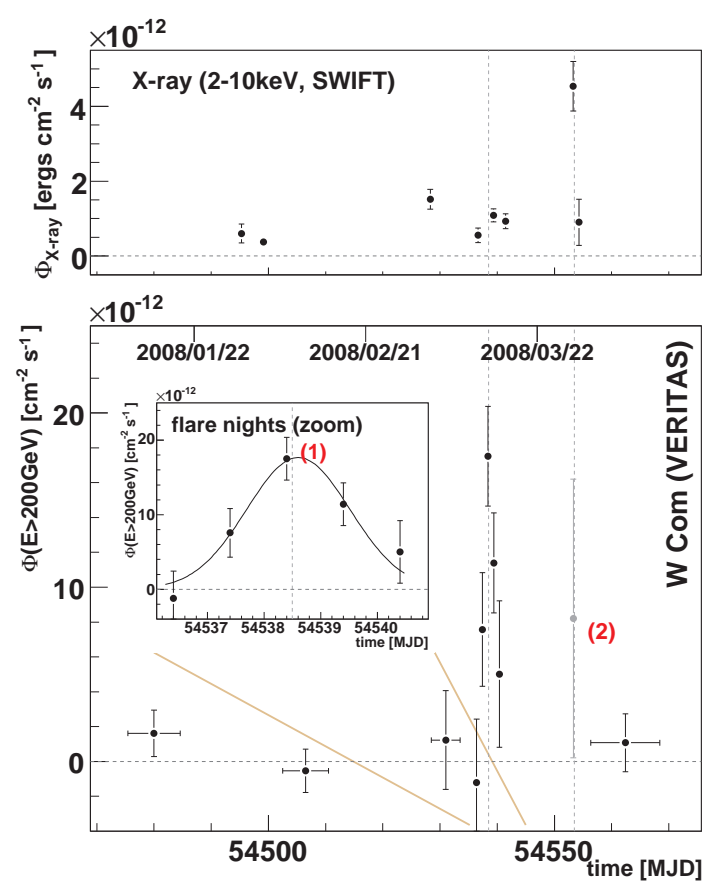

FIGURE 13. The upper panel shows the light curve of the integral photon flux from W Comae as observed by VERITAS in March, 2008 [21], [24]. Each point corresponds to one night of observation. The upper panel shows the X-ray flux as measured by Swift. This was the first detection of a signal from this IBL AGN. A second stronger flare was seen in June, 2008 [23]. The source is only detected when flaring.

\section{Multiwavelength Coverage: $10^{8} \mathrm{to} 10^{27} \mathrm{~Hz}$}

To probe the astrophysics of the sources it is particularly valuable to make correlated observations across the electromagnetic spectrum. The TeV observations, coming at the extreme end of the spectrum, are unique and generally stretch the models to their limits. A number of campaigns have been organized around the $\mathrm{TeV}$ observations; these typically involve radio, infrared, optical, $\mathrm{X}$-ray and gamma-ray observatories; an example of the results of one such campaign (on the most variable AGN, Markarian 421) is shown in Figure 14 and 15 (D. Horan, private communication). Of particular value in the study of blazers are correlated observations with hard X-ray observatories.

The organization of multi-wavelength campaigns is particularly difficult because of the difficulties of scheduling diverse instruments in space and on the ground. It is complicated by the different cultures that prevail in the different wavebands. The production of papers based on such campaigns requires the patience of Job. Since many TeV physicists do not have a background in classical astronomy, involvement in such campaigns has the added advantage of increased aware- 


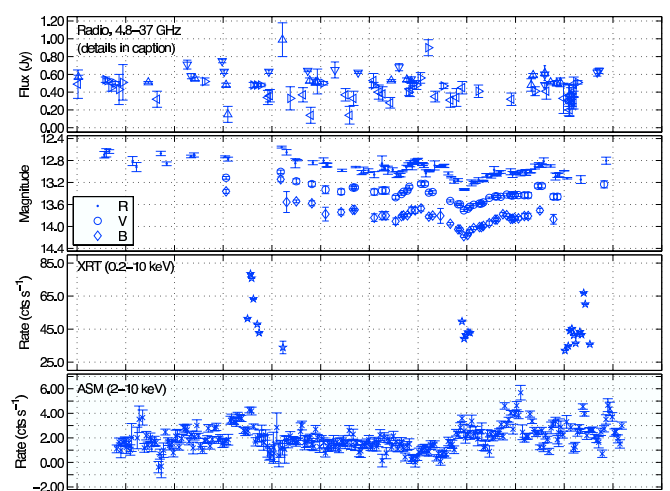

FIGURE 14. Observations of Markarian 421 taken in an extensive multi-wavelength campaign from November, 2005 to June, 2006, which was organized by the Whipple gamma-ray group. Data were taken on four radio telescopes and thirteen optical telescopes (as well as two X-ray satellites and the Whipple 10m telescope) (Figure 15). Variability was found at almost all wavelengths. The radio data are plotted in the top panel: $4.8 \mathrm{GHz}, 8 \mathrm{GHz}, 14.5 \mathrm{GHz}$ and $37 \mathrm{GHz}$. The optical data are combined from the different observatories in three color bands.

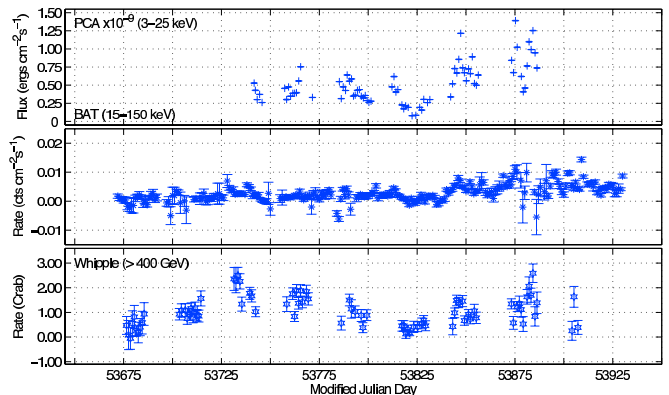

FIGURE 15. The $\mathrm{X}$-ray and $\mathrm{TeV}$ gamma-ray data points over the same time period as in Figure 14 . The X-ray points come from RXTE (ASM) and Swift (BAT). The gamma-ray points are from the Whipple $10 \mathrm{~m}$ gamma-ray telescope with threshold $400 \mathrm{GeV}$; each data point is the flux recorded on that night of the observing campaign.

ness in other astronomical disciplines. It also increases awareness of $\mathrm{TeV}$ astronomy in the wider astronomical community.

\section{Source Density}

Source confusion was not considered a problem for $\mathrm{TeV}$ observatories until recently when the unexpected density and complexity (Figure 16) [25] of sources indicated that as the sensitivity of observatories increased, the probability of finding more than one source within

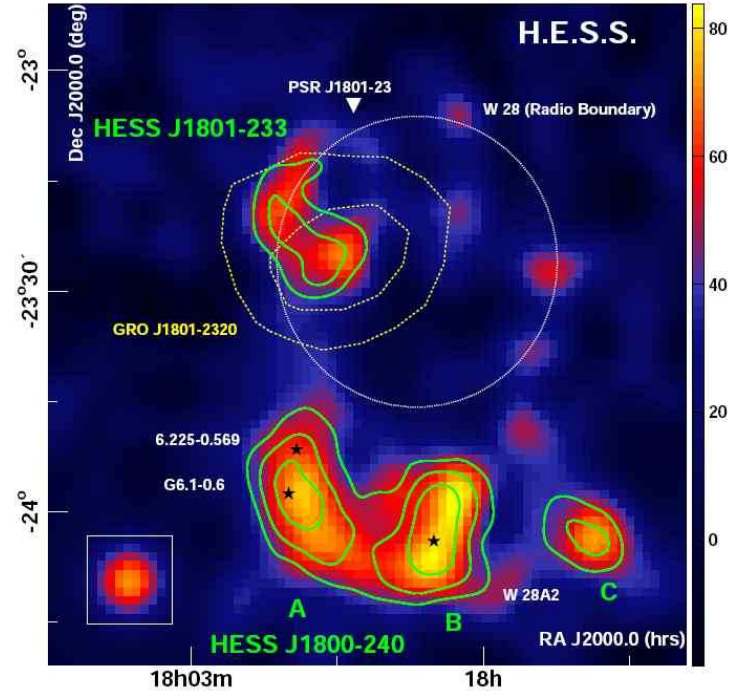

FIGURE 16. The TeV gamma-ray complex image near W28 as seen by H.E.S.S. [25]. The solid contours of the gammaray excess are at significance levels of 4,5 , and $6 \sigma$. The dashed circle depicts the approximate radio boundary of the SNR W 28. There are at least four gamma-ray sources in this map.

the limited field of view of the detectors was definitely finite. While this was not too unexpected close to the Galactic Plane it was somewhat unexpected when it involved extragalactic sources (Figure 17).

Much of the sky has not yet been systematically surveyed; it is important to reexamine archival data in cases where a new source is detected in an apparently empty field.

\section{PROGRESS TO DATE}

Those who have been involved in, or who have followed, the development of this field cannot help but be pleased at the progress made to date. Some of this progress was predictable but generally I believe most observers have been amazed (and pleased) at how much has been achieved.

A brief list of the highpoints of what we now know about the TeV sky must include:

- Multitude of Sources: initially it was supposed that the TeV sky would be dominated by a small number of sources, probably supernovae remnants, in which hadrons would be accelerated by some process and gamma rays would be produced in the decay of neutral pions created in the collision of the hadrons with gas; this is clearly not the case with many diverse categories of sources and with electron progenitors 


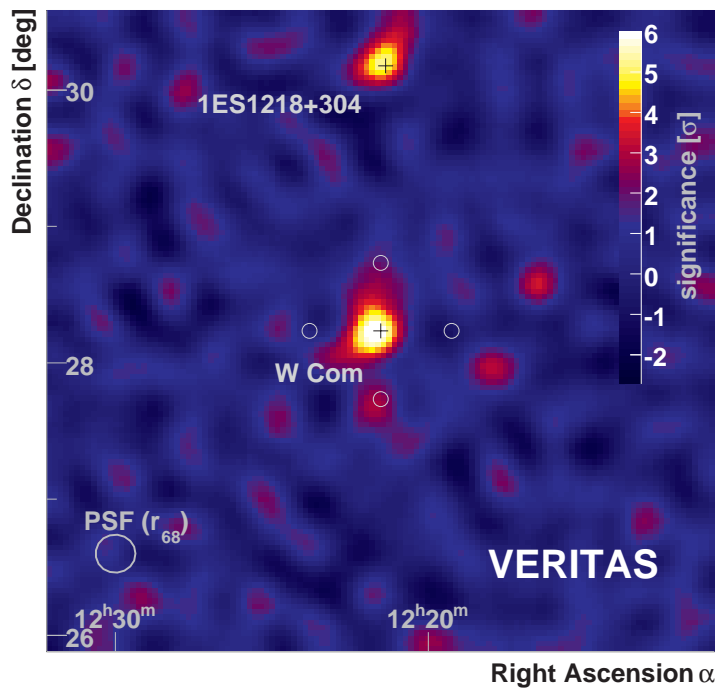

FIGURE 17. VERITAS sky map of significances centered on the position of W Comae (cross) [24]. Although 1ES1218+304 $(\mathrm{z}=0.182)$ had previously been detected, it was again apparent in the W Comae $(\mathrm{z}=0.0 .080)$ observations and illustrates the sensitivity to off-axis sources and the density in the sky of AGN.

apparently playing a major role.

- Supernovae Remnants: as expected, gamma-ray emission is seen from some supernovae remnants of the shell variety but they are by no means the dominant sources in the Galaxy; the conventional theory of origin of the cosmic radiation is not substantiated by the $\mathrm{TeV}$ gamma-ray observations made to date.

- The $100 \mathrm{MeV}$ Connection: an early assumption was that the TeV sky would be a weak extension of the rich $100 \mathrm{MeV}$ sky revealed by SAS-2, COS-B, and EGRET; in fact the TeV sky has been shown to be an entirely different animal and the large EGRET catalog is seen to be a poor predictor of $\mathrm{TeV}$ emission. Hard X-ray emission correlates better with $\mathrm{TeV}$ emission and is an indication that ComptonSynchrotron models with electron progenitors can explain the emission in many sources.

- Unidentified Sources: the existence of the so-called "dark sources" highlights the fact that the TeV sky has unique features that are not apparently duplicated in other wavebands. Based on their spatial distribution it appears that they are mostly Galactic sources.

- Spectra: the spectra of Galactic TeV sources is, in general, much harder than expected; with a few notable exceptions the most interesting discoveries have come, not at the lowest threshold energies, but at the medium energies where the telescopes have the maximum flux sensitivity.

- Extended Sources: since the IACT is particularly sensitive for the detection of point sources, it was not surprising that the first sources detected were all point-like. That many of the new Galactic sources have significant angular extent has been a pleasant surprise and has opened new avenues of astrophysical investigation with comparison of source maps at different wavelengths. The angular resolution of the IACT is surprisingly good and exceeds that found to date at other gamma-ray energies.

- Extragalactic Sources: the number of extragalactic sources is larger than expected; clearly cosmic ray acceleration is not just a Galactic phenomenon. The $\mathrm{TeV}$ blazer catalog is the most complete catalog of any category of source and can be expected to grow since even with current sensitivity only a small fraction of the sky has been explored. In contrast much of the nearby rich Galactic sky has been scanned with high sensitivity.

- Transients: one of the great strengths of the groundbased techniques is their large collection area and hence high sensitivity to transient emission. The extragalactic sources exhibit much more variability than the sources found thus far in the Galactic Plane. The time-scale of variations in these sources is much shorter than expected and opens the possibility of testing physical laws as well as pointing to some extraordinary astrophysical processes.

- Absorption: there is less absorption (by photonphoton pair production) in intergalactic space than had been expected; the observable gamma-ray universe is therefore larger than the pessimistic early predictions would have suggested.

- Moonlight: the advent of arrays of imaging detectors with stable trigger systems permit the observation of gamma-ray sources under moonlight with only marginally reduced sensitivity. Although generally the time around full moon is avoided, the traditional number of useful observing hours can be increased by $30-40 \%$.

\section{SOURCE CATALOGS}

It is not possible to summarize all the observational results in a single publication although a good attempt can be found in [5]. In this field catalogs are difficult because sources are detected by a variety of instruments and new sources are constantly being added. Unlike space missions, ground-based experiments do not have sharp turnon and turn-off dates so that it is hard to say when a catalog is complete. A useful catalog of the many sources found by the H.E.S.S. observatory can be found on their 
Webpage [26] but naturally this does not include sources detected by other groups and hence is biased towards the Southern Hemisphere. Another useful web catalog is that maintained by Deirdre Horan and Scott Wakely [27] which only lists those sources that have been accepted for publication. To give some measure of the progress in the field and to provide a bench mark for checking future progress, the generally accepted sources and their vital parameters are gathered here in a series of Tables that are loosely based on the above catalogs.

These are divided into five tables: Extragalactic sources (Table 2), Supernovae Remnants (Table 3), Pulsar Wind Nebulae (Table 4), Milagro Sources (Table 5), Binaries/Miscellaneous (Table 6), and Dark Sources (Table 8). All the sources are designated by their Right Ascension (2000) and Declination (2000) coordinates which have been rounded; a TeV prefix is attached to each source irrespective of its discoverer.

Table 2 lists the catalog of known extragalactic sources; not only is it one of the largest source categories but it is also one of the least ambiguous and the most homogeneous. The 24 sources are listed in terms of their catalog and common name, their redshift (which determines their place in the table), their flux at $1 \mathrm{TeV}$ (usually variable), their power law spectral index, their classification and the discovery group and date. For the sources listed in this and subsequent tables, the original references can be found in [5]. Clearly the predominant sources are BL Lac objects whose SEDs are peaked towards higher frequencies. In all of these objects the TeV emission is associated with the jets.

The distinction between the objects listed in Tables 3 and 4 is not always clear cut. Classical shell supernovae remnants are relatively easy to model and to understand. Objects like those found in the vicinity of W28 are obviously related and their subdivision into distinct sources is somewhat arbitrary. There is particular interest in objects in which the supernovae seems to be interacting with nearby molecular clouds; these are the best candidates for hadronic acceleration and interaction. Pulsar Wind Nebulae are more difficult to model and less likely to be hadronic sources. The pulsar is generally off center and the structure is complex; the Crab Nebula is clearly an exception.

The Milagro sources (Table 5) are all detected at energies in excess of $20 \mathrm{TeV}$. Only one has been also detected at lower energies by IACT observatories. Since they are all extended and have no clear counterparts, they represent a population that is quite distinct from the sources listed in the other tables.

Table 6 lists a number of diverse but important identified sources. Among these are the four binaries (whose parameters are also listed in Table 7) [29]. They do not fit into any standard class but these objects probably represent only a small sample of the TeV binary emitters.
In many ways the unidentified sources listed in Table 8 are most interesting since they might represent an entirely new class of object. They are located very close to the Galactic Plane; they seem to be concentrated towards the Galactic Center; they are all extended; all have very flat spectra. They may turn out to be Pulsar Wind Nebulae that for some reason are obscured at other wavelengths; this is the least exciting possibility. Other suggestions are that they might be unusual supernovae remnants, pulsar wind nebulae, giant molecular clouds, stellar clusters with powerful winds or Gamma Ray Burst remnants.

\section{OUTLOOK}

\section{Retrospective}

Nearly fifty years ago the real pioneers of TeV astronomy, Chudakov and Zatsepin in the U.S.S.R. and Jelley and Porter in the British Isles, made their first brave venture into this now rich field of $\mathrm{TeV}$ gamma-ray astronomy; it was a major leap of faith since it was truly terra incognita. When these early experiments were planned, there were no known $100 \mathrm{Mev}$ sources; indeed in 1960 there were no known X-ray sources and thus the known astrophysical electromagnetic spectrum beyond the earth effectively ended in the near ultraviolet. Although the observation of the cosmic radiation was a clear indication that high energy particles must be accelerated somewhere, commonsense would have suggested that since the cosmic particles were observed to have a steeply falling energy spectrum, it was unlikely that the first high energy sources would be apparent at the high gamma-ray energies where the fluxes would be very low. As early as 1962 it was pointed out that photon-photon absorption might be a serious limitation for $\mathrm{TeV}$ gamma-ray astronomy ([28]); fortunately the optical cosmic photon density was overestimated and the gamma-ray horizon was not as near as these gloomy predictions indicated. The few models of TeV source intensity that were proposed considered only pion production in hadron collisions and were speculative at best.

In fact these early pioneers were either woefully ignorant of the astrophysics or extremely optimistic; either way they had to have great self-confidence to feel that they had the ability to beat the odds!

TeV Gamma-ray Astronomy is now a mature science with cutting edge instruments, mature observatories, dedicated and experienced adherents, and catalogs of diverse sources. The question might then be asked why this discipline took so long to develop for, unlike its counterparts in the X-ray and $100 \mathrm{MeV}$ gamma-ray bands, it did not have to await the development of space 
TABLE 2. Extragalactic Sources

\begin{tabular}{|c|c|c|c|c|c|c|}
\hline Catalog Name & Common Name & Redshift & $\begin{array}{r}\text { Flux at } \mathbf{1 ~ T e V} \\
10^{-12} \mathrm{~cm}^{-2} \mathrm{~s}^{-1} \mathrm{TeV}^{-1}\end{array}$ & Index & Classification & $\begin{array}{r}\text { Discovery } \\
\text { (Group/Date }\end{array}$ \\
\hline TeV1231+124 & M87 & 0.00436 & 1 & 2.9 & FRI & HEGRA/2003 \\
\hline TeV1104+382 & Markarian 421 & 0.031 & $12-97$ & $2.4-3.1$ & HBL & Whipple/1992 \\
\hline TeV1654+398 & Markarian 501 & 0.034 & $0.5-100$ & $1.9-2.3$ & $\mathrm{HBL}$ & Whipple/1996 \\
\hline TeV2347+517 & 1ES2344+514 & 0.044 & $1-5$ & $2.3-2.5$ & $\mathrm{HBL}$ & Whipple/1998 \\
\hline TeV1136+702 & Markarian 180 & 0.045 & 0.9 & 3.3 & HBL & MAGIC/2006 \\
\hline TeV2000+651 & 1ES1959+650 & 0.048 & $4-120$ & $2.7-2.8$ & HBL & Tel.Arr./2000 \\
\hline TeV0551-323 & PKS0548-323 & 0.067 & 0.3 & 2.8 & $\mathrm{HBL}$ & H.E.S.S./2007 \\
\hline $\mathrm{TeV} 2203+423$ & BL Lacertae & 0.069 & 0.3 & 3.6 & LBL & Crimea/2001 \\
\hline TeV2009-488 & PKS2005-489 & 0.071 & 0.2 & 4 & HBL & H.E.S.S./2005 \\
\hline TeV0152+017 & RGB J0152+017 & 0.080 & & 2.95 & HBL & H.E.S.S./2008 \\
\hline TeV1221+283 & W Comae & 0.102 & & 3.81 & IBL & VERITAS/2008 \\
\hline TeV2159-302 & PKS2155-304 & 0.117 & $2-3$ & $2.3-2.5$ & HBL & Durham/1999 \\
\hline TeV1429+427 & H1426+428 & 0.129 & $1-2$ & $2.6-3.7$ & HBL & Whipple/2002 \\
\hline TeV0809+524 & $1 \mathrm{ES} 0806+524$ & 0.138 & & & HBL & VERITAS/2008 \\
\hline $\mathrm{TeV} 0233+203$ & $1 \mathrm{ES} 0229+200$ & 0.140 & 0.62 & 2.5 & HBL & H.E.S.S./2006 \\
\hline TeV2359-306 & H2356-309 & 0.165 & 0.3 & 3.1 & $\mathrm{HBL}$ & H.E.S.S./2006 \\
\hline TeV1221+302 & 1ES1218+304 & 0.182 & 1.3 & 3.0 & HBL & MAGIC/2006 \\
\hline TeV1103-232 & 1ES1101-232 & 0.186 & 0.4 & 2.9 & HBL & H.E.S.S./2007 \\
\hline TeV0349-115 & 1ES0347-121 & 0.188 & 0.45 & 3.1 & HBL & H.E.S.S./2007 \\
\hline TeV1015+495 & 1ES1011+496 & 0.212 & 0.3 & 4.0 & HBL & MAGIC/2007 \\
\hline TeV1556+112 & PG1553+113 & $0.3-04$ & $0.1-0.2$ & 4.0 & HBL & H.E.S.S./2006 \\
\hline TeV0219+425 & $3 \mathrm{C} 66 \mathrm{a}$ & 0.444 & & & IBL & Crimea/1998 \\
\hline TeV1256-058 & $3 \mathrm{C} 279$ & 0.536 & - & 4.1 & FSRQ & MAGIC/2008 \\
\hline TeV0716+714 & S50716+714 & $?$ & & & $\mathrm{HBL}$ & MAGIC/2008 \\
\hline
\end{tabular}

TABLE 3. Supernova Remnants

\begin{tabular}{llrrrrl}
\hline $\begin{array}{r}\text { Object } \\
\text { Catalog }\end{array}$ & Common Name & $\mathbf{l}_{I I}$ & $\mathbf{b}_{I I}$ & Type & $\begin{array}{r}\text { Distance } \\
\text { kpc }\end{array}$ & $\begin{array}{r}\text { Discovery } \\
\text { Group/Date }\end{array}$ \\
\hline TeV0616+225 & IC443 & 189.03 & 2.90 & Shell (PWN?) & 1.5 & MAGIC/2007 \\
TeV0852-463 & R0852-4622 & 266.28 & 1.24 & Shell & 10.2 & H.E.S.S./2005 \\
TeV1442-625 & RCW 86 & & & Shell & 1 & H.E.S.S./2007 \\
TeV1714-398 & RX J1713.7-3946 & 347.28 & 0.38 & Shell & 1 & CANGAROO/2001 \\
TeV1714-382 & CTB37B & 348.65 & -0.38 & 10.2 & SNR & H.E.S.S./2006 \\
TeV1714-385 & CTB37A & 348.39 & 0.11 & 10.3 & SNR & H.E.S.S./2008 \\
TeV1747-282 & G0.9+0.1 & 0.87 & 0.08 & SNR (PWN?) & 8.5 & H.E.S.S./2005 \\
TeV1802-233 & W28 & 6.66 & 0.27 & Shell & 2 & H.E.S.S./2008 \\
TeV1833-105 & G21.5-0.9 & 21.5 & -0.7 & Shell (PWN?) & 4 & H.E.S.S./2008 \\
TeV1846-027 & Kes 75 & 29.9 & 0.0 & SNR (PWN?) & $6-19$ & H.E.S.S./2008 \\
TeV2323+588 & Cassiopeia A & 111.73 & -2.1 & SNR & 3.4 & HEGRA/2001 \\
\hline
\end{tabular}

TABLE 4. Pulsar Wind Nebulae

\begin{tabular}{llrrrrr}
\hline $\begin{array}{r}\text { Object } \\
\text { Catalog }\end{array}$ & \multicolumn{1}{c}{ Common Name } & Association & $\mathbf{l}_{I I}$ & $\mathbf{b}_{I I}$ & $\begin{array}{r}\text { Distance } \\
\text { kpc }\end{array}$ & $\begin{array}{r}\text { Discovery } \\
\text { Group/Date }\end{array}$ \\
\hline TeV0535+220 & Crab Nebula & M1 & 184.56 & -5.78 & 2 & Whipple/1989 \\
TeV0835-463 & Vela X & & 263.91 & -3.01 & 0.29 & H.E.S.S./2006 \\
TeV1418-610 & Kookaburra Rabbit & G313.3+0.1? & 313.25 & 0.15 & 5.6 & H.E.S.S./2006 \\
TeV1420-607 & Kookaburra Pulsar & P1420-6048 & 313.56 & 0.27 & 5.6 & H.E.S.S./2006 \\
TeV1514-592 & & MSH 15-52 & 320.33 & -1.19 & 5.21 & H.E.S.S./2005 \\
TeV1641-465 & & G338.3-0.0 & 338.32 & -0.02 & 8.6 & H.E.S.S./2005 \\
TeV1718-385 & & 348.83 & -0.49 & 4.2 & H.E.S.S./2005 \\
TeV1811-193 & & PSR J1809-1917 & 11.18 & -0.09 & 3.7 & H.E.S.S./2007 \\
TeV1826-138 & & PSR J1826-1334 & 17.82 & -0.74 & 3.9 & H.E.S.S./2005 \\
TeV1913+102 & & PSR J1913+1011 & 44.39 & -0.07 & & H.E.S.S./2007 \\
\hline
\end{tabular}


TABLE 5. Milagro Sources

\begin{tabular}{rlrrlr}
\hline $\begin{array}{r}\text { Object } \\
\text { Catalog }\end{array}$ & Common Name & $\mathbf{l}_{I I}$ & $\mathbf{b}_{I I}$ & $\begin{array}{r}\text { Size } \\
\text { degrees }\end{array}$ & $\begin{array}{r}\text { Discovery } \\
\text { Group/Date }\end{array}$ \\
\hline TeV1908+060 & MGRO J1908+06 & 40.16 & -0.93 & 0.5 & Milagro/2007 \\
TeVJ2019+37 & MGRO J2019+37 & 75.11 & 0.54 & $1.1 \pm 0.5$ & Milagro/2007 \\
TeV2031+41 & MGRO J2031+41 & 79.72 & 0.94 & $3.0 \pm 0.9$ & Milagro/2007 \\
\hline
\end{tabular}

TABLE 6. Binaries and Odd and Ends

\begin{tabular}{llrrlrl}
$\begin{array}{r}\text { Object } \\
\text { Catalog }\end{array}$ & Common Name & $\mathbf{l}_{I I}$ & $\mathbf{b}_{I I}$ & & $\begin{array}{r}\text { Type } \\
\text { Distance } \\
\text { kpc }\end{array}$ & $\begin{array}{r}\text { Discovery } \\
\text { Group/Date }\end{array}$ \\
\hline TeV0240+612 & LSI +61 303 & 135.68 & 1.09 & Binary & 2 & MAGIC/2006 \\
TeV0633+058 & & 205.66 & -1.44 & Binary? & 1.6 & H.E.S.S./2007 \\
TeV1023-575 & Westerlund 2 & 284.19 & -0.39 & Stellar cluster & 8 & H.E.S.S./2007 \\
TeV1302-638 & PSR 1259-63 & 304.19 & -0.99 & Binary & 1.5 & H.E.S.S./2005 \\
TeV1746-290 & Galactic Center & 359.95 & -0.05 & Black Hole & 8.5 & CANGAROO/2004 \\
TeV1746-290 & Galactic Ridge & 359.95 & -0.05 & Diffuse Source & 8.5 & H.E.S.S./2006 \\
TeV1759-240 & W28C & 5.7 & -0.1 & Unknown & $2-4$ & H.E.S.S./2008 \\
TeV1800-240 & W28B & 5.90 & -0.36 & Molecular Cloud & $2-4$ & H.E.S.S./2008 \\
TeV1800-240 & W28A & 6.14 & -0.63 & Molecular Cloud & $2-4$ & H.E.S.S./2008 \\
TeV1826-149 & LS 5039 & 16.88 & -1.29 & Binary & 2.5 & H.E.S.S./2005 \\
TeV1958+352 & Cyg X-1 & 71.3 & 3.1 & XRB & 2.2 & MAGIC/2007 \\
\hline
\end{tabular}

TABLE 7. Binary Source Parameters ([29]

\begin{tabular}{lllll}
\hline \multicolumn{1}{c}{ Source/Parameter } & PSR B1259-69 & LSI+61 303 & LS5039 & Cygnus X-1 \\
\hline Type & B2Ve+NS & BOVe+NS & O6.5+BH? & O9.7Iab+BH \\
Distance (kpc) & 1.5 & $2.0 \pm 0.2$ & 2.5 & $2.2 \pm 0.2$ \\
Periodicity (days) & 1,237 & 25.5 & 3.9 & 5.6 \\
VHE & Regular $?$ & Irregular & Regular & Transient \\
Observatory & H.E.S.S. & MAGIC, VERITAS & H.E.S.S. & MAGIC \\
Radio Period & $48 \mathrm{~ms}, 3.4$ yr & $26.5 \mathrm{~d}, 4 \mathrm{yr}$ & Steady & Steady \\
L (X-rays) $\times 10^{33} \mathrm{erg} / \mathrm{s}$ & $0.3-6$ & $3-9$ & $5-50$ & 10,000 \\
L (VHE gamma)x $10^{33} \mathrm{erg} / \mathrm{s}$ & 2.3 & 8 & 7.8 & 12 \\
Index (VHE) & $2.7 \pm 0.2$ & $2.6 \pm 0.2$ & $2.06 \pm 0.05$ & $3.2 \pm 0.6$ \\
EGRET Source & - & 3EG J0241+6103 & $3 \mathrm{EG} \mathrm{J1824-1514}$ & - \\
\hline
\end{tabular}


TABLE 8. Unidentified (Dark) Sources

\begin{tabular}{lrrrrr}
\hline Object//Catalog & $\mathbf{l}_{I I}$ & $\mathbf{b}_{I I}$ & Index & $\begin{array}{r}\text { Association } \\
\text { (Possible) }\end{array}$ & $\begin{array}{r}\text { Discovery } \\
\text { Group/Date }\end{array}$ \\
\hline TeV1303-632 & 304.24 & -0.36 & 2.4 & & H.E.S.S./2005 \\
TeV1428-608 & 314.41 & -0.14 & 2.16 & & H.E.S.S./2008 \\
TeV1614-518 & 331.52 & -0.58 & 2.46 & & H.E.S.S./2005 \\
TeV1616-509 & 332.39 & -0.14 & & PSR J1617-5055 & H.E.S.S./2005 \\
TeV1626-490 & 334.77 & 0.05 & 2.18 & & H.E.S.S./2008 \\
TeV1632-478 & 336.38 & 0.19 & 2.12 & I16320-4751 & H.E.S.S./2006 \\
TeV1635-473 & 337.11 & 0.22 & 2.4 & I16358-4726 & H.E.S.S./2006 \\
TeV1703-420 & 344.30 & -0.18 & 2.1 & P1702-4128 & H.E.S.S./2006 \\
TeV1708-410 & 345.68 & -0.47 & 2.46 & & H.E.S.S./2006 \\
TeV1732-347 & 353.57 & -0.62 & 2.3 & & H.E.S.S./2008 \\
TeV1745-304 & 358.71 & -0.64 & 1.82 & & H.E.S.S./2006 \\
TeV1805-217 & 8.40 & -0.03 & 2.7 & G8.7-0.1 & H.E.S.S./2005 \\
TeV1809-194 & 10.92 & 0.08 & & PSR J1809-1917 & H.E.S.S./2007 \\
TeV1814-178 & 12.81 & -0.03 & 2.1 & G12.82-0.02 & H.E.S.S./2005 \\
TeV1835-088 & 23.24 & -0.32 & 2.5 & W41/G23.3-0.3 & H.E.S.S./2005 \\
TeV1838-070 & 25.18 & -0.11 & 2.27 & G25.5+0.0 & H.E.S.S./2005 \\
TeV1841-056 & 26.80 & -0.2 & 2.4 & & H.E.S.S./2008 \\
TeV1857+027 & 35.96 & -0.06 & 2.39 & PSR1856+025 & H.E.S.S./2008 \\
TeV1858+021 & 35.58 & -0.58 & 2.1 & & H.E.S.S./2008 \\
TeV2032+415 & 80.25 & 1.07 & 1.9 & Cyg OB2 & HEGRA/2002 \\
\hline
\end{tabular}

technology. By 1980 the concept for a new generation of telescope had already been proposed ([30];[31];[32]; [33])

It may be that this second generation of astronomers were not imaginative enough or were too conservative to exploit the possibilities of these energy bands. But the slow progress must also be at least partially because the ground-based Cherenkov technique was not easy to categorize and thus it was difficult for funding agencies to fit it into their normal modes of support for astrophysical research. At a time when "gamma-ray astronomy" was synonymous with space astronomy (and support therefore assumed to come from national space agencies such as NASA), there was no natural conduit for serious funding. It was not really high energy physics, it was not space astronomy, it was not traditional cosmic ray astronomy and it required the dark high remote sites traditionally associated with optical astronomy. Even the Smithsonian Astrophysical Observatory, which, with internal funding, had been prepared to gamble on such ventures in the lush days of the sixties withdrew support in 1976 when it was apparent that the early results were not promising enough for NASA to support as providing useful complementary observations to the space missions.

The first real impetus to develop the IACT in the USA came about as a direct result of the activity of neutrino astronomers who had a high profile and ready constituency of support from high energy physicists. In fact it was largely pressure from the neutrino astronomy community to build a major underwater telescope that led the High Energy Division of the U.S. Department of
Energy to fund the pioneer effort in IACT at the Whipple Observatory in 1982. The thinking here was that if it could be demonstrated that there were no sources of $\mathrm{TeV}$ gamma rays detected with this more sensitive, but relatively inexpensive, technique, then there would be little justification for the construction of the much more expensive neutrino telescopes. The slow development of the IACT was thus justification for the agencies to delay the large investment necessary for the construction of large neutrino telescopes; high energy physics funding could then be reserved for the perceived more interesting area of high energy particle research at accelerators. The subsequent success of the IACT observatories has been a major impetus for the construction of the new generation of neutrino telescopes even though there is still not strong evidence that the progenitor particles in most TeV gamma-ray sources are hadrons and therefore likely neutrino producers.

\section{Perspective}

The present plethora of sources at $\mathrm{TeV}$ energies must mean that the prospects for fruitful research are bright for the coming epoch. If the number of participants means anything, then one cannot but be impressed by the fact that hundreds of scientists (if one is to judge by the list of authors) have migrated into this field; this is far in excess of the numbers at any previous epoch. 
While much has been done, there is still much to be done. Among the potential sources yet to be detected are:

- Pulsars (more than one)

- Starburst Galaxies

- Dark Matter

- Auger Source Counterparts

- Gamma Ray Bursts

Although there is more than enough work to do with the present instrumentation it is important, and indeed inevitable, that some effort is devoted to the development of the technology necessary for a new generation of telescopes. It is easy to envisage the extension of the IACT by simply multiplying the number of telescopes; the difficulty is to do so economically. An N-fold increase in the number of telescopes (and approximately in cost) only results in an increase in sensitivity by a factor of $\mathrm{N}^{0.5}$. In the absence of a major technical breakthrough, then we are entering into an era of extremely expensive ground-based observatories; in fact the costs will begin to be in the same ballpark as the cost of building space telescopes.

There is not a single driving scientific justification for this major upgrade in sensitivity but rather a desire to do better in all the areas currently being explored [6]. In the current, rather gloomy, economic climate it will be a brave effort to seek funding in excess of a hundred million dollars or euros without a single critical scientific imperative (like the nature of dark matter, the existence of dark energy or the meaning of life!). However no such single mission objective existed for Fermi (GLAST) either.

Two such efforts of IACT construction are now under consideration: the largely European CTA which aims to scale up the existing arrays and hopes to achieve savings by mass production, and the somewhat more innovative US AGIS which would attempt to develop a new approach to telescope and camera design. Some might argue that all resources could be pooled so that one major observatory could be built with the maximum sensitivity. However while it makes sense to have as much cooperation as possible between such observatories, there is a strong argument to be made for at least two independent observatories using different technologies (apart from the obvious one of needing two to fully cover both hemispheres). It is very important in extending a discipline into a new region of parameter space that marginal and threshold detections can be confirmed and that systematic effects be identified. AGILE and Fermi are in this happy situation. Given the wide range of phenomena that might be observed it is unlikely that the observation requirements can be satisfied by a single instrument without introducing serious compromises in its design. Also since some four decades of the electromagnetic are avail- able, it may be sensible to concentrate and optimize on particular bands. The large fields of view that are optimum for the study of extended Galactic sources are generally wasteful for the study of point sources like AGN. The mapping of supernovae remnants at high energies requires instruments with very large collection areas and high angular resolution.

The ongoing observations using the IACT will be complemented by the continued operation and extension of the ground-based particle arrays. These have already demonstrated that they are capable of detecting $\mathrm{TeV}$ sources; long integrations are possible because of their high duty cycle. They are particularly sensitive to extended sources. Although the anticipated detection of transient sources has not materialized, the completion of HAWC (this symposium) by the Milagro group at a high elevation site in Mexico will open new possibilities in this regard.

Given the fiscal realities of 2008 it will not be surprising if, for the next few years, the ground-based IACT community must rely on incremental improvements to existing observatories, rather than order of magnitude scaling up to a new generation of observatories. It is somewhat disappointing that despite the success of the IACT, no dramatic improvement in detection technique has been proposed. A moratorium imposed by the bleak economy may be advantageous in that the results obtained with the current observatories can be thoroughly considered, new technologies may be taken advantage of, and innovative ideas for new detection strategies fostered.

In the coming decade gamma-ray astronomy in the $\mathrm{GeV}$ regime will surely be dominated by AGILE and Fermi; complementary observations by upgraded versions of the current ground-based observatories at energies in excess of $100 \mathrm{GeV}$ where the IACT is most sensitive will extend the scientific impact of these missions and form a firm basis for new facilities, both in space and on the ground.

\section{ACKNOWLEDGMENTS}

My research is supported by grants from the U.S. Department of Energy, the U.S. National Science Foundation, and the Smithsonian Institution. Participation in the Heidelberg Gamma-ray 2008 Symposium was possible using personal funding sources. I am grateful to my VERITAS colleagues (M.Beilicke, P.Cogan, D.Horan, P.Fortin, M. Hui) for supplying figures ahead of publication and to J.Perkins for reading the manuscript. 


\section{REFERENCES}

1. Weekes, T.C., "TeV Gamma-ray Astrophysics", Heidelberg Workshop, October, 1994, Space Science Reviews, 75, 1 (1996)

2. Weekes, T.C., "High Energy Gamma Ray Astronomy", Heidelberg International Symposium, June, 2000, AIP Conference Proceedings, 558, 15, (2000)

3. Hinton, J., Proceedings of 30th International Cosmic Ray Conference, Merida, Mexico, July 2007, (in press), astro-ph0712.3352 (2007)

4. De Angelis, A., Mansutti, O., Persic, M., Il Nuovo Cimento, (in press) astro-ph 0712.0315 (2008)

5. Aharonian, F., Buckley, J., Kifune, T., Sinnis, G., J.Phys.G: Nucl.Part.Phys. (in press) (2008)

6. Buckley, J., et al., APS White Paper, astro-ph:0810.0444 (2008)

7. Abdo, A.A. et al., ApJL, 643, L53 (2007)

8. Cawley, M.F, et al. 19th International Cosmic Ray Conference, La Jolla, California) 1, 131 (1985)

9. Weekes, T.C., et al., ApJ, 342, 379, (1989)

10. Fazio, G.G., Helmken, H.F., Rieke, G.H., Weekes, T.C., ApJL, 154, L83 (1968)

11. Otte, A.N., MAGIC Collaboration, Proceedings of 30th International Cosmic Ray Conference, Merida, Mexico, July, 2007 , astro-ph 0712.1386 (2007)

12. Aharonian, F. et al., A\&A, 464, 235 (2007)

13. Djannati-Atai, A., H.E.S.S. Collaboration, Proceedings of 30th International Cosmic Ray Conference, Merida, Mexico, July, 2007, Astro-ph 0710-2418, (2007)

14. The Astronomer's Telegram, 1415, 1 (2008)

15. Cogan, P., VERITAS Collaboration (this Symposium) (2008)

16. Aharonian, F. et al., ApJL, 664, L71 (2007)

17. Hui, M., VERITAS Collaboration, (this Symposium) (2008)

18. Y. I. Neshpor et al., Astronomy Letters 24 (1998) 134

19. The Astronomer's Telegram, 1753, 1 (2008)

20. Fortin, P., VERITAS Collaboration (this Symposium) (2008)

21. The Astronomer's Telegram, 1422, 1 (2008)

22. Albert, J. et al., 2006, ApJ, 642, L119.

23. The Astronomer's Telegram, 1565, 1 (2008)

24. Beilicke, M., VERITAS Collaboration (this Symposium) (2008)

25. Aharonian, F. et al., A\&A, 481, 401 (2008)

26. http://www.mpihd.mpg.de/hfm/HESS/pages/home/sources/

27. Horan, D., Wakely, S., http://tevcat.uchicago.edu/

28. Nikishov, A.J., Sov. Phys.-JETP, 14, 393 (1962)

29. Parades, J.M., astro-ph 0803-1097 (2008)

30. Jelley, J.V., Porter, N.A., M.N.R.A.S. 4, 275 (1963) 1964

31. Hill., D.A., Porter, N.A., Nature, 191, 690 (1960)

32. Grindlay, J. et al. ApJL, 559, 100 (1996)

33. Weekes, T.C., Turver, K.E., Proc.12th ESLAB Symp.(Frascati), 279 (1977) 